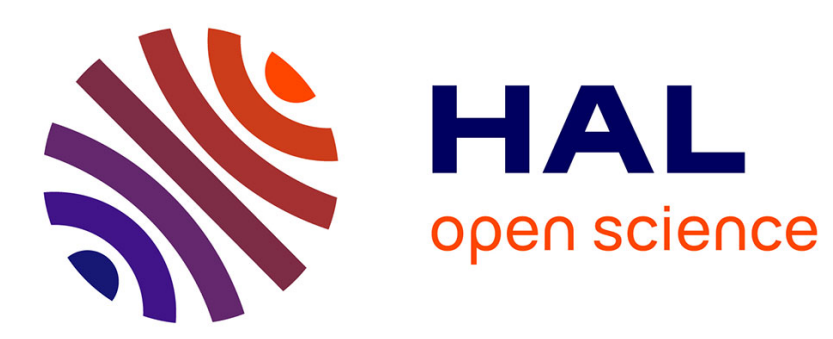

\title{
La géographie rurale "quantitative et théorique": bilan d'une décennie
}

\author{
Violette Rey, Marie-Claire Robic
}

\section{To cite this version:}

Violette Rey, Marie-Claire Robic. La géographie rurale "quantitative et théorique" : bilan d'une décennie. Annales de géographie, 1983, 92 (511), pp.305-330. 10.3406/geo.1983.20190 . hal-02884402

\section{HAL Id: hal-02884402 \\ https://hal.science/hal-02884402}

Submitted on 29 Jun 2020

HAL is a multi-disciplinary open access archive for the deposit and dissemination of scientific research documents, whether they are published or not. The documents may come from teaching and research institutions in France or abroad, or from public or private research centers.
L'archive ouverte pluridisciplinaire HAL, est destinée au dépôt et à la diffusion de documents scientifiques de niveau recherche, publiés ou non, émanant des établissements d'enseignement et de recherche français ou étrangers, des laboratoires publics ou privés. 


\section{La géographie rurale "quantitative et théorique" : bilan d'une} décennie

Violette Rey, Marie-Claire Robic

\section{Citer ce document / Cite this document :}

Rey Violette, Robic Marie-Claire. La géographie rurale "quantitative et théorique" : bilan d'une décennie . In: Annales de Géographie, t. 92, n511, 1983. pp. 305-330;

doi : https://doi.org/10.3406/geo.1983.20190

https://www.persee.fr/doc/geo_0003-4010_1983_num_92_511_20190

Fichier pdf généré le 24/02/2020 


\title{
Résumé
}

Au seuil de la modélisation systémique, les travaux de géographie rurale écrits dans une perspective « quantitative et théorique » sont peu nombreux. Leur unité ne tient pas au choix de sujets particuliers ou de techniques préférentielles, mais à une orientation de recherche. Celle-ci valorise l'explicitation, la mesure et vérification, les constructions théoriques. Les résultats tiennent compte des connaissances acquises, ce qui conduit à un effort particulier de théorisation dans le domaine agricole déjà très étudié, et à une exploration de la spécificité territoriale de cette nouvelle entité qu'est l'espace rural. Ce qui lie les deux domaines, c'est la préoccupation de la composante territoriale des phénomènes et un effort de conceptualisation proprement géographique. Ces démarches s'orientent vers les modèles spatiaux les plus connus, mais non retenus par la géographie rurale classique. La décennie 1980 s'ouvre sur une vision plus claire des points à approfondir.

\begin{abstract}
On the fringe of systemic model-making, works done in rural geography with a theoric and quantitative approach are rather scarce. Their common factor has nothing to do with the choice of particular topics or techniques, but comes from the orientation of the research itself. The stress is put on explanations, quantitative measurements - and their checking - and theoretical models. Results take previous knowledge into account, which leads first to a special theorizing effort in the already much-studied agricultural field, and, second, to an attempt to define a new spatial entity, the so-called rural space. Both fields are linked by the concern for the spatial component of the phenomena and an effort of geographical conceptualization. These processes lead to the most well-known models, so far neglected by classic rural geographers. The eighties open out on a clearer view of the points that need to be deepened.
\end{abstract}




\title{
La géographie rurale "quantitative et théorique": bilan d'une décennie
}

\author{
V. Rey, \\ Université de Paris I \\ M.-C. Robic, \\ L.A. 142 du C.N.R.S.
}

Etablir un bilan de l'apport de l'informatique à la géographie urale? C'est un problème de départ classique, où il faut faire la part des effets liés aux contraintes de l'instrument, aux changements de l'objet étudié, aux mutations d'ensemble d'une collectivité scientifique. L'instrument risque de disparaître en tant que tel ici. Nous avons en effet privilégié deux points de vue: celui de l'ambition avouce de nombre de tenants de la géographie "quantitative et théorique", utilisateurs de l'informatique; celui des contributions spécifiques à la géographie rurale, l'une des branches les plus anciennes et les plus productives en chercheurs et en recherches accumulées.

Nous avons examiné les travaux pour situer leur apport dans la double perspective d'une "nouvelle géographie" et du contexte de sa réalisation ${ }^{1}$. Le modèle de référence, vécu comme une autre

1. Il était vain de penser établir un inventaire exhaustif des travaux pertinents, surtout en ce domaine où abondent les publications ronéotées ou à diffusion restreinte. Si la définition de critères de sélection dans le domaine quantitatif est assez claire à établir. la délimitation du champ théorique est plus floue. De fait, les travaux retenus ont plus systématiquement un caractère quantitatif qu'une orientation théorique. La sélection bibliographique est opératoire; elle n'exprime pas de jugement de valeur par rapport aux autres recherches rurales.

Nous avons retenu les travaux reposant pour une part sur un outil informatique (comme une base de données informatisée), ou sur des calculs du niveau de la statistique bivariée, ou de la statistique inférentielle, et les travaux dont les titres se réfèrent explicitement à la théorie. L'expression "quantitative" équivaut dans le texte à "quantitative et théorique". 
pratique, est un modèle complexe de scientificité, à visée théorique ou formalisatrice, qui dépasse la seule quantification. Il tend à rechercher des règles ou des lois, plutôt qu'à identifier des cas "uniques", à se référer à un modèle déductif plutôt qu'à user de l'induction généralisante, à développer une recherche transparente c'est-à-dire qui apporte ses preuves ou ses moyens de vérification, qui explicite les démarches et repose sur un langage non ambigu. A cet égard, ce travail nous a montré que la rupture est parfois plus projetée que consommée, qu'un certain bricolage n'est pas exclu, et que la nouveauté technique ne s'associe pas automatiquement à novation ou perfectionnement conceptuel.

Le contexte? La contribution "quantitative" ne peut s'interpréter sans tenir compte de la combinaison particulièrement contraignante de l'observation, dans un temps court pour des innovations technologiques complexes (1970-1980), de travaux inclus dans une phase longue de désintérêt des jeunes chercheurs pour l'espace rural. Ce désintérêt reflète une subordination économique, politique et idéologique de la campagne au profit de la ville, qui s'est traduite par un appauvrissement relatif des moyens de connaissance (chercheurs, crédits). De plus, parallèlement à cette position de repli, il fallait rendre compte d'une transformation de l'objet: l'espace rural s'est transformé non seulement dans sa base productive traditionnelle (l'agriculture), mais encore dans l'ensemble de ses fonctions. L'outillage conceptuel hérité, les interrogations classiques, voire les modèles interprétatifs existants pouvaient-ils servir de points de départ à des recherches sur ces changements? Pour accentuer la complexité du contexte, l'irruption de contre-courants contredisant les valeurs modernistes et technocratiques a progressivement revalorisé ce domaine rural mal connu dans ses transformations.

$\mathrm{Au}$ total, c'est sur un effectif très réduit d'auteurs que cette expérimentation a fonctionné. Aussi les explications des choix de thèmes, de démarches, d'outils, ont une portée limitée par le faible nombre des travaux: les aléas habituels aux innovations n'existentils pas ici aussi ? Ni bilan, par rapport à l'ensemble des thèmes de la géographic rurale, ni évaluation de l'apport "quantitatif» à la problématique de chaque auteur, les pages qui suivent sont exploratoires.

Comme l'ensemble des géographes quantitativistes, les ruralistes sont passés par trois phases: une première, au début des années 1970, d'exploration et d'intuition de la diversité des pistes théoriques et méthodologiques; une seconde d'uexercices" d'apprentissage, surtout centrée sur les outils descriptifs de l'analyse des données (analyses factorielles et classifications); la troisième, actuelle, aborde des recherches beaucoup plus sophistiquées: les travaux intègrent la constitution d'une information 
spécifique et l'explicitation de questions touchant à des problèmes spatiaux à des méthodologies complexes, moins strictement descriptives, articulant fichiers informatiques, traitements statistiques, graphique et cartographie automatique. Sur ce bref laps de temps, nous avons cru saisir une certaine dualité: les recherches en matière agricole sont marquées d'un certain raffinement de la tradition, tandis que le domaine d'étude de l'espace rural non agricole est plus novateur dans ses thèmes. Notre présentation sera fondée sur ces deux aspects, suivie d'une synthèse de la contribution "quantitative" à la géographie rurale.

1. Géographie quantitative et théorique dans la géographie agraire et agricole, raffinement d'une tradition?

Trois quarts de sic̀cle d'une géographie française largement attentive au monde des campagnes et aux travaux agraires dominent la production géographique rurale contemporaine. A la différence de la sociologie tardivement ouverte à l'orientation rurale, la géographie a donc acquis une connaissance déjà très approfondie des formes et des interrelations agricoles. Effet du patrimoine d'une part, prééminence croissante des mécanismes économiques et de l'aiguillon technique de l'autre, les deux ont orienté l'analyse contemporaine des réalités agraires davantage en direction des "mutations et permanences" que des remises en question théoriques. La très petite fraction de géographes "quantitativistes" qui ont travaillé dans le domaine agraire ne se distingue pas sensiblement par les démarches retenues: les typologies, les réinterprétations régionales de thèmes classiques comme le vignoble, la différenciation agricole, le foncier l'emportent sur l'utilisation de certains modèles vedettes de la nouvelle géographie. Par ailleurs, les travaux de géographie agricole tropicale n'ont pas encore de résultats dans l'optique quantitativiste, moins sans doute par un dédain de cette problématique que par nécessité préalable de constituer les banques de données. L'outil informatique associé à la télédétection en milieu tropical est déjà devenu quasi indispensable (GILG, 1978).

Le classement des thèmes agricoles en fonction de la plus ou moins grande fréquence des études «quantitatives» qui leur sont consacrées montre un clivage qui est en relation inverse avec le degré de territorialité des thèmes eux-mêmes.

1.1. Les domaines de l'habitat, du paysage agraire, les rapports entre les activités agricoles et les milieux physiques

Ce sont ceux dans lesquels les «progrès" géographiques sont actuellement les plus limités. 
La non-reprise de l'étude de l'habitat rural par les quantitativistes est significative d'une attitude de pause face à un thème et d'une faible inclination pour l'expérimentation géographique formelle de la part des ruralistes. La question de la répartition de l'habitat n'a qu'exceptionnellement fait l'objet de traitement systématique en terme de répartition probabiliste, vérifiant le degré de spécificité régionale de divers types de semis de peuplement (Baker, 1968, sur la Beauce et la Gâtine tourangelle; Chiffre, 1969).

Les paysages ne sont ni oubliés ni négligés (des thèses récentes consacrent des analyses attentives aux paysages ibériques ou de la France de l'Ouest). Mais un certain état d'achèvement de la connaissance géographique classique a été atteint. Il est significatif que ce soient les tropicalistes, les bio-géographes et les agronomes, ceux qui, à des titres divers, étaient les plus dégagés des concepts paysagistes géographiques, qui ont appliqué des techniques quantitatives à l'étude des paysages. Leur "méconnaissance» relative du paysage les a conduit à s'y pencher au moment où apparaissait, sur le marché de l'information, l'immense et nouveau domaine de données fourni par la télédétection. La masse documentaire énorme exigeait une nouvelle démarche d'analyse. Appuyé sur des chaînes méthodologiques voisines - analyse d'images de couverture de photo aérienne, constitution de fichiers décodant les images, traitements graphiques et statistiques des données mises en fichiers, cartographies le plus souvent automatiques - l'effort a porté sur la détermination des unités élémentaires d'observation, leur niveau de regroupement et sur la sélection des variables prises en compte. Le concept de "paysage intégré » a été mis au point avec, dans les trois cas, le souci de fournir une "trame paysagique" opérationnelle pour des projets d'aménagement agricoles et ruraux. Si de tels travaux ont permis d'étendre rapidement la couverture des paysages agraires connus, ils débouchent surtout sur des classifications physionomiques naturalistes de niveaux d'homogénéité et offrent des bases analytiques à la description de l'espace. Les concepts d'" agrosystème ", de géosystème..., traités en termes d'analyse de système, en sont sans doute à la transition entre élaboration théorique et utilisation opérationnelle (Bertrand, 1978; Brossard et Wieber, 1980).

L'analyse des rapports entre l'agriculture et le milieu physique est un domaine classique où les géographes quantitativistes, à l'image de la collectivité générale des ruralistes, ont peu progressé. "L'idéologie" du progrès technique réduisant la contrainte physique à un facteur résiduel n'y est pas étrangère. En outre, l'orientation pratique prépondérante en termes d'analyses de données sur des unités territoriales assez vastes s'accommodait mal d'une intégra- 
tion de données physiques soit trop générales pour être fécondes, soit non pertinentes a ces échelles (Guermond, 1979, et l'évaluation pédologique en cinq classes des unités de la France de l'Ouest). Néanmoins la question des rapports entre les conditions physiques et l'activité agricole peut, en l'état des connaissances, être posée en termes plus étroits de "niveaux de contrainte", de "degrés de liberté ", la perspective "théorique et quantitative" offrant alors au moins deux voies possibles. L'une, prolongeant des recherches analytiques antérieures (du type facteurs physiques des crus viticoles), passe premièrement par des séries de mesures extrêmement fines, deuxièmement par la recherche d'équations qui exprimeraient la forme des fonctions liant les observations (Béguin, 1974, et l'ajustement entre les précipitations et les rendements au Maroc). L'autre consiste à définir les niveaux moyens de rente différentielle susceptibles à un moment donné d'être obtenus en divers lieux. Cette piste a de fortes implications non seulement en termes de théorie économique, mais aussi de théorie géographique puisqu'elle concerne la définition des différenciations territoriales; en outre elle relève tout autant d'une problématique pratique de géographie applicable puisque les institutions ont besoin d'une telle connaissance pour établir le "Répertoire de la valeur des terres agricoles».

H. Béguin (1976) a proposé une esquisse méthodologique suivie d'une application sommaire de l'évaluation de l'influence résiduelle des milieux géographiques dans l'efficacité de l'aménagement de la vallée du Sebou au Maroc; la préoccupation est évidente chez V. Rey (1982), mais la mise en forme systématique d'une telle entreprise, qui passe nécessairement par une quantification puis des tests, reste à faire, de même que la confrontation entre les notions de rendement économique et de rendement écologique.

\subsection{L'analyse fonctionnelle des systèmes agricoles}

Elle a été un des domaines privilégiés des géographes «quantitativistes ».

A notre avis, trois séries de facteurs ont orienté les travaux dans cette direction. La mutation agricole française "imposait " cette étude fonctionnelle à tous les géographes; le puissant appareil statistique agricole facilitait l'exploration systématique des chiffres jusqu'à un niveau territorial fin; le principal appareillage alors utilisé - celui de l'analyse des données - était assez bien adapté aux projets de descriptions systématiques du fonctionnement spatioéconomique de l'agriculture. Analyses factorielles et classifications automatiques ont permis de dégager les associations fortes entre variables, d'identifier des combinaisons territoriales spécifiques, de 
proposer des types, voire d'aborder l'explication statistique de la distribution par d'autres variables; les traitements graphiques de données, fichiers-images et matrices ordonnables, ont également été utilisés dans la même optique.

Utilisation $d u$ sol et typologies agricoles sont les deux grands domaines de prédilection pour l'emploi de ces traitements.

L'étude de l'utilisation $d u$ sol appuyée sur l'exploitation de données statistiques a une solide tradition dans la géographie française: les travaux cartographiques d'A. Perpillou, établis sur un modèle de référence empirique, le fameux terroir-type national; la thèse de J. Klatzmann, à sa manière précurseur des traitements graphiques de données. En outre, les économistes ont parallèlement repris la question de la localisation des cultures (Ruillière), ont utilisé l'analyse factorielle des correspondances (Guigou) pour observer la forme du déplacement des cultures et la restructuration de l'espace agricole ${ }^{2}$. Y. Guermond de même a mis au point un algorithme pour traiter des combinaisons culturales en France et de leur évolution. A ce stade, il y a un gain incontestable: outre la rapidité (théorique) d'exécution, ces analyses peuvent garder un large éventail de données, établir des profils complexes, complets et explicites de l'utilisation du sol dans chaque unité territoriale d'observation, les classer en types. On retrouve fréquemment de telles analyses dans les différentes thèses faisant usage des outils quantitatifs (Auriac, 1979; Béguin, 1975; Calmès, 1978; Rey, 1980, etc.). On peut considérer qu'il s'agit alors d'un usage "standard » de l'analyse de données; une image structurelle de base, la trame de l'utilisation du sol, est rapidement établie, comparable dans son rôle de connaissance physionomique initiale aux classements qu'établissent les géographes urbains sur les profils de villes ou de quartiers selon les catégories socio-professionnelles ou les catégories d'activités économiques.

Les typologies agricoles rassemblent un éventail de phénomènes plus large que les classements d'utilisation du sol; elles soulèvent des problèmes plus complexes, en fonction de la place accordée au traitement mathématique et en fonction de la nature des éléments sur lesquels sont établies les typologies. Les travaux de J. Bonnamour et Ch. Gillette (1980) sur les types d'agriculture en France, à partir des normes proposées par J. Kostrowicki, sont dans une position originale. A la suite de travaux qui ont progressivement

2. Perpillou A.. 1952, "Essai d'établissement d'une carte de l'utilisation du sol en France", Acta geographica, $\mathrm{n}^{\circ} 18,110-115$.

Klatzmann J., 1955, Localisation des cultures et des productions animales en France, Paris, Imprimeric Nationale.

Rulliere G., 1956, Localisation et rythmes de l'activite agricole, Paris, A. Colin. Villars.

Guigou J.-L., 1972, Théorie économique et transformation de l'espace agricole, Paris, Gauthicr- 
défini d'un point de vue géographique les concepts agricoles, les auteurs ont sélectionné un système de variables composé de plusieurs sous-systèmes. La construction logique est à proprement parler un modèle de référence d'où sont déduits les types d'agriculture ${ }^{3}$. Dans cette démarche, l'intervention "quantitative " se borne aux calculs intermédiaires et à l'affectation de chaque profil agricole territorial à l'un des profils-types; l'outil trie, il ne calcule aucune corrélation, n'opère aucun rapprochement entre des variables de distribution statistique similaire mais appartenant à des sous-systèmes différents. N'y a-t-il pas une sous-exploitation de l'information contenue dans les données? Les autres typologies agricoles font beaucoup plus appel à la puissance des algorithmes mathématiques pour classer les éléments du réel et les interpréter en termes de types agricoles.

Les typologies agricoles recouvrent deux sortes d'objets, et à ce titre la discussion sur les variables sélectionnées ne présente pas la même signification. Les unes opèrent sur les agents agricoles, les exploitations (Lenco, 1975,... en général les économistes); dans ce cas le strict respect d'une collecte de données qui soient fonctionnelles par rapport aux agents se justifie pleinement; la typologie mathématique doit ensuite établir un classement "objectif " des agents (c'est-à-dire non entaché de rapprochements intuitifs liés à l'expérience individuclle du chercheur). Les autres typologies opèrent sur des espaces; elles veulent classer les espaces en fonction de la spécificité de combinaisons agricoles et pour des fins diverses (connaissance fondamentale, découpage d'aménagement...); dans ce cas, l'introduction de variables d'environnement avec les variables de fonctionnement est alors souvent pratiquée sans que cela soit automatiquement préjudiciable à l'intelligence du réel, tout au contraire.

\subsection{Les situations foncières agricoles et les processus d'inno- vation}

Ils constituent les deux autres domaines où l'approche quantitativiste a amorcé des analyses, moins nombreuses que pour les typologies, mais sans doute avec une plus grande diversité de procédure. La maîtrise du traitement en chaîne des analyses factorielles et des classifications permet de décrire les formes et les rythmes de l'inégale concentration foncière: on peut substituer à la comparaison d'indices de Gini, très synthétiques, la comparaison de profils de répartition des exploitations selon les catégories de taille; l'analyse de régression appliquée aux évolutions de chaque catégorie

3. Toutes les variables sélectionnées et réparties en sous-systèmes sont a priori découpées en cinq classes de valeur: des profils-types sont ensuite déterminés selon les associations de classe de valeur de chaque sous-système. 
permet de cerner le rôle spécifique joué par certaines d'entre elles dans le changement global (Brunet, Le Rouzic, 1979, Rey, 1980). Les cartes nationales des prix de la terre ont été largement explicitées par les analyses de régression multiples (Moindrot, 1978, Vaylet ${ }^{4}$ ), qui ont permis d'identifier des facteurs explicatifs "stables". Un tel acquis relatif au marché foncier est l'occasion de souligner les difficultés que l'on rencontre dès que l'on veut expliquer certains phénomènes, même élémentaires. Autant la géographie du prix s'avère facile à interpréter ainsi, autant celle du volume des superficies vendues achoppe: le modèle de régression est beaucoup plus difficile à établir, ce qui atteste de l'hétérogénéité des facteurs, plus grande que pour une "fonction" prix. Et pourtant le volume des ventes est au moins aussi important pour l'intelligence des changements géographiques (Rey, 1980).

En matière d'analyse des processus d'innovation, les ruralistes plutôt quantitativistes se sont paradoxalement peu référé aux démarches spatiales formalisées par Hågerstrand. Seul Y. Guermond (1975) a analysé cette question avec une problématique de mesure spatiale et a proposé un modèle de simulation pour la diffusion de l'équipement de stabulations libres en Haute-Normandie.

\section{Exploration de nouveaux thèmes en géographie rurale?}

La différenciation socio-économique de l'espace rural est l'un des champs privilégiés de l'analyse "quantitative et théorique». Cette orientation résulte de la rencontre des techniques éprouvées de l'analyse des données, bien adaptées à la description de la diversité et des types, et d'une interrogation ancienne sur les types de campagnes, revitalisée par les actuelles transformations sociologiques et fonctionnelles (dont témoigne, dans le vocabulaire, l'adoption de l'expression espace rural).

En l'absence d'un important patrimoine de recherches antérieures sur l'espace rural, les thèmes dominants de la géographie "quantitative et théorique " résultent souvent moins de problématiques théoriques initiales que de choix empiriques liés à l'aspect spectaculaire du changement à étudier, à la facilité d'utilisation des données de recensement et à des demandes sociales. Difficultés méthodologiques, volonté de démarquage ou simple aléa lié au faible effectif de chercheurs? De nombreuses questions ne sont que peu traitées: exode rural, découpages de l'espace, stratégies des

4. Vaylet J.-I.., 1973, Le Marché foncier des terres agricoles en France, 1964-1972, aspects fondamentaux et évolution récente, S.C.A.F.R., Paris, $140 \mathrm{p}$. 
acteurs, signification du changement social, perception de l'espace rural, etc.

Peu de modèles spatiaux ont été importés, à la différence de la géographie urbaine, qui a découvert ceux de "l'écologie factorielle" par exemple. Les études se veulent même souvent strictement descriptives, ce qui poursuit une (illusoire) tradition. Cependant des modèles interprétatifs socio-économiques peuvent intervenir explicitement dans la mise en forme de la recherche. Généralement, les mêmes questions reviennent, "semi-théoriques" et classiques comme l'effet des variations de densité, ou plus neuves, comme celle de l'articulation, dans les processus de changement, entre activités agricoles et non agricoles. Des différences profondes se révèlent toutefois dans la complexité de l'élaboration intellectuelle, dans le soin apporté à la démonstration et dans l'attention accordée à la spatialisation des phénomènes. Par l'étude des processus spatiotemporels du changement, et par celle des formes d'organisation de l'espace rural, les recherches récentes les plus novatrices tentent de découvrir des régularités et de dégager, à partir des formes de territorialité et de la géométrie des réseaux, la logique des localisations et des divisions spatiales.

\subsection{Les composantes de la diversité socio-économique}

La plupart des analyses multivariées qui explorent la diversité de l'espace rural se contentent, dans un premier temps, d'une sorte de «mise à plat» descriptive, de lecture parfois difficile, des composantes dégagées par analyse factorielle. Une démarche plus théorisée revient à interpréter la différenciation au sein de l'espace rural dans le cadre d'un modèle global socio-économique. C'est par exemple le cas de R. Chapuis (1979), pour qui c'est une exigence de cohérence que de retrouver, en fin d'étude, la distinction majeure qui a présidé au choix des variables analysées, sélectionnées au nom d'un modèle d' "urbanisation des campagnes" inspiré d'E. Juillard. Enfin, quelques études visent à répondre à des questions particulières, telles: "le niveau de densité" ou la "vulnérabilité" — à définir et à mesurer quantitativement - déterminent-ils la constitution de types d'espace?

Avec la référence France entière et le niveau des "espaces ruraux départementaux", Calmès et al. (1978) ont pris le parti d'étudier l'espace rural français moins à travers sa spécificité sociologique et productive agricole que par son caractère d'espace à faible densité d'occupation et dans sa dimension d'espace de consommation. C'est à cette détermination que se rapportent des analyses globales destinées - une fois l'ampleur et l'originalité du "fait rural" 
mesurées - à cerner la diversité des espaces ruraux et à définir les principales interactions (association, exclusion, indépendance) entre leurs caractéristiques économiques, sociales, démographiques, de résidence, d'équipement et d'encadrement urbain. L'un des objectifs majeurs est, outre la recherche des règles de distribution, de discerner si les multiples composantes de la diversité rurale sont cohérentes avec les grands découpages de l'espace français.

Les résultats acquis et les hypothèses explicatives, partiellement testées au cours de l'ouvrage, ont été plus systématiquement étudiés dans des travaux ultérieurs où les auteurs ont mobilisé d'autres informations et d'autres techniques statistiques plus adaptées à leur objectif de compréhension des localisations et des structures spatiales (Calmès et al., 1979, 1981; Durand-Dastès, 1981). C'est le cas notamment de l'étude de la diversité fonctionnelle, observée par le biais des catégories socio-professionnelles des résidents et des actifs au lieu de travail. Les auteurs constatent une faible spécificité des espaces ruraux par rapport à leur département. F. Durand-Dastès (1981) a confirmé et enrichi cette constatation en poursuivant l'étude de la distribution des actifs non agricoles à l'aide d'un modèle explicatif liant espace rural et espace urbain, et de tests garantissant le caractère plausible des régularités spatiales observées. Il retrouve dans l'espace rural les oppositions principales entre catégories socio-professionnelles détectées dans l'espace urbain (catégories salariées et autres d'une part; groupes de salariés socialement valorisés et autres salariés d'autre part). L'étude comparée des agencements géographiques des catégories socio-professionnelles (en plages uniformes, en répartition ponctuelle, etc.) confirme l'existence dans l'espace rural et dans l'espace urbain départemental de fortes cohésions spatiales (mesurées par l'auto-corrélation), qui reflètent les classiques contrastes économiques français. La plus grande "valeur ajoutée" de ce travail réside toutefois dans la révélation des nuances, très difficilement lisibles sur des cartes analytiques: elles suggèrent des répartitions spatiales différenciées qui renvoient à des logiques de décision spatiale du travail.

A d'autres niveaux d'analyse et avec d'autres espaces de référence, de nombreux auteurs géographes et non géographes (tel Piatier, 1979) ont exploré la diversité de l'espace rural pour aboutir à des typologies d'espaces élémentaires ou pour dégager les éléments structurants de l'espace rural. La variété des problématiques et des milieux induit une grande variété de résultats. Cependant des régularités se dessinent. Généralement, apparaît comme premier axe différenciateur l'opposition entre espaces très agricoles et les autres, à la fois plus peuplés, mieux équipés, et disposant d'activités plus variées: l'effet de taille (effet structurel et 
artefact des analyses) domine souvent, tandis que les autres facteurs de différenciation du milieu rural apparaissent en second lieu. A ce niveau, le choix des variables étudiées, largement dépendant de considérations implicites, détermine les distinctions. A été particulièrement élu le "dynamisme", sous ses variantes démographique, économique (Aitchison et al., 1981), financière (Limouzin, 1975 et 1979), ou agricole (Chapuis, 1979 et 1982).

\subsection{Les transformations de l'espace rural: le changement social,} fonctionnel et de l'usage du sol

Par rapport à la gamme des études rurales sur le changement, les quelques recherches "quantitatives et théoriques" ont comme point commun d'être traversées en filigrane par la question de la "diversification" de la base économique, de la société rurale et des utilisations du sol; en corollaire, elles s'interrogent sur une éventuelle tendance à l'homogénéisation de l'espace rural. Mais les problématiques et les espaces de référence restent trop disparates pour donner lieu à des réponses synthétiques. De plus, si les techniques d'analyse spatio-temporelles se sont améliorées, l'appréhension des processus temporels reste délicate en raison des contraintes d'hypothèses des modèles mathématiques utilisables, de la faible longueur des séries étudiées et de difficultés techniques pour intégrer le temps et l'espace. Quant aux essais d'interprétation, ils sont particulièrement peu nombreux dans ce domaine.

A l'échelle de la France entière, n'a été abordée quantitativement que la diversification des activités (N. Mathieu, dans La France de P. Pinchemel (1980), dont l'étude porte sur les départements et les trois secteurs économiques en une comparaison 1954 et 1975 qui utilise la méthode de Weaver). Le changement social, thème-roi, objet d'A.T.P., étudié pour l'ensemble des villes ${ }^{5}$, a surtout fait l'objet d'une série d'études à moyenne échelle sur le LanguedocRoussillon. Qu'ils proviennent de la comparaison d'analyses factorielles des "états" de 1954, 1962 et 1968 (Auriac et al., 1974), de l'étude des liens statistiques entre indicateurs d'évolution intercensale (Auriac et al., 1975) ou de l'étude des "trajectoires" relatives des cantons et des catégories socio-professionnelles dans une analyse conjointe des quatre recensements de 1954 à 1975 (Bernard, 1981), les résultats sont convergents. Ils mettent en évidence un processus de diversification sociale dans les campagnes qui, avec la réduction du nombre et de la proportion des agriculteurs, avec la montée des «cols blancs » et le maintien des effectifs ouvriers, tend à banaliser

5. Pumain D., Saint-Julien T., 1978, Les Dimensions du changement urbain, Paris, éd. du C.N.R.S., 202 p. 
globalement l'espace rural par rapport aux villes. S'agit-il pour autant d'une homogénéisation? Il semble - mais l'analyse mériterait d'être poussée - que l'émergence de nouvelles combinaisons socio-professionnelles (où pèse surtout l'inégale diffusion des cadres supérieurs et divers «cols blancs») produise une nouvelle diversité spatiale.

Les catégories socio-professionnelles discriminantes ont change, et surtout entre 1962 et 1968; l'évolution numérique des agriculteurs ne paraît pas dépendre de celle des autres catégories (Auriac et al., 1975); les évolutions des cantons ont été essentiellement liées à celle des catégories non agricoles (Bernard, 1981). La dissociation entre évolutions d'effectifs agricoles et non agricoles se retrouve dans l'échantillon cantonal étudié par Aitchison et al. (1981).

Les mutations spectaculaires de l'espace péri-urbain et leurs enjeux économiques expliquent la faveur dont il a bénéficié. Le faible nombre de recherches recensées ici (sur Martigues, Metz et Montpellier), comparé à la surabondance des travaux en la matière (cf. Berger et al., 1980) ne souligne que mieux l'aspect partiel des contributions des quantitativistes à la connaissance des processus de péri-urbanisation. Peu nombreux, ils se sont de plus restreints ( 2 sur 3) à l'étude statistique du plus visible (et du plus aisément quantifiable?): la transformation de l'utilisation du sol, en privilégiant l'extension du bâti. Encore faut-il souligner leur commun effort de formalisation et de cohérence conceptuelle et technique, ainsi que les innovations méthodologiques et l'apport factuel du plus achevé d'entre eux surtout (groupe Dupont, 1981a): l'ensemble - hétérogène certes - constitue un groupe d'études exemplaire de l'état de la géographie "quantitative et théorique", à la rencontre de l'urbain et du rural.

\subsection{Les disparités face à l'équipement}

Très peu abordée dans les études rurales classiques, agrocentrées et productivistes (cf. Berger et al., 1975), la question de l'équipement des campagnes est devenue l'un des sujets les mieux traités de la recherche "quantitative et théorique". La sensibilisation des ruraux aux inégalités dont ils étaient victimes et les craintes face à une dégradation des espaces ruraux au-delà d'un certain seuil de dépeuplement ont impulsé ce mouvement de recherche. Les carences de l'information statistique en la matière (malgré l'enquête communale du ministère de l'Agriculture), la lourdeur des relevés à opérer (recours au Bottin téléphonique) et les difficultés particulières du traitement d'objets qui sont des éléments ponctuels ou linéaires de réseaux - où interviennent densité, géométrie, hiérarchie, fluidité - rendent ces recherches particulière- 
ment ardues. C'est peut-être ce qui explique le caractère tardif de leur apparition, sous cette dernière forme surtout (de réseau), malgré l'existence de nombreuses références dans l'arsenal théorique et technique de l'analyse spatiale, et de la géographie de la distribution des communes et des services (Berry, 1971).

Quoi qu'il en soit, c'est la variété et la richesse des perspectives théoriques et pratiques qui caractérisent ce domaine, étudié en général à l'échelle de la France entière. Nous pouvons orienter son analyse en fonction de quelques interrogations dominantes, issues tout autant de questions anciennes que de résultats controversés ou récurrents.

\subsubsection{Equipement et densité de population}

Les travaux systématiques menés sur la France des faibles densités (Bontron et al., 1977) concluent à la réalité du handicap d'équipement subi dans les zones de faible peuplement, qui sont aussi des zones a forte prépondérance rurale. Par contre, cartes et calculs de corrélation linéaire révèlent qu'un grand nombre de problèmes fréquemment attribués à la faible densité ne sont pas généralisables à l'ensemble de la zone ou ne sont pas associés au niveau de peuplement. Plus que les indicateurs de gestion de l'espace, d'activité économique et de vie sociale, les indicateurs d'équipement montrent une réelle homogénéité des espaces peu peuplés. Un échantillonnage au niveau des cantons permet d'affiner ces conclusions et de mieux discerner les traductions spatiales de types de structure et d'évolution (Aitchison et al., 1981).

C'est sur cette même question des disparités d'équipement que s'est penché le groupe de géographie rurale de Paris VII. Leurs premières études (Calmès et al., 1978) ont souligné les très fortes inégalités départementales d'équipement commercial, la régularité la plus manifeste étant la liaison entre la qualité de l'équipement et les effectifs de population communale. Les auteurs (Calmès et al., 1979 et 1981) ont perfectionné cette analyse en interprétant les disparités de l'offre commerciale grâce à un modèle des localisations qui oppose à une logique spatiale (tendant à fixer les commerces en un seul lieu pour couvrir tout le marché), une logique "de population" (qui tend à la disperser en direction de la clientèle). La comparaison de la densité commerciale et des distances entre communes équipées (des espaces ruraux départementaux entre eux et avec les seules communes urbaines) permet de mettre à jour, à nouveau, régularités nationales et spécificités locales ou régionales. Elle confirme la relation directe entre la taille des maillages commerciaux ruraux et les densités de population rurales ou totales d'une part, les trames communales d'ensemble d'autre part. L'étude de la configuration des réseaux commerciaux (selon la méthode d'analyse des semis de points dite du "plus 
proche voisinage", appliquée au Gers et à quinze départementstests), montre des distributions spatiales plutôt régulières que concentrées ou aléatoires (la "logique de population" prévaudrait donc) «régularisant» en quelque sorte le semis de base constitué par l'ensemble des communes, sur lequel se moule celui des communes équipées.

\subsubsection{Equipements et seuils de population}

La question des seuils de population a été soulevée souvent dans sa version "critique", c'est-à-dire comme seuil de peuplement (par exemple niveau de densité) au-delà duquel les risques de désertification sont patents. Si théoriquement l'existence de seuils est concevable, il n'apparaît, semble-t-il, pas de limite de densité séparant des comportements démographiques ruraux: des mécanismes de compensation jouent probablement (Bronton et al., 1977).

Par contre, des études précises sur la distribution des commerces et des services révèlent des régularités: au-delà d'un apparent continuum, ceux-ci se localisent bien selon certaines normes de hiérarchie des centres qui les accueillent, tout en présentant des variations systématiques que seules des études comparatives à multiples paramètres peuvent mettre à jour (cf. études de B. Berry, 1971). Les études qui se réfèrent aux théories de la centralité ont accumulé maintenant une somme considérable de résultats locaux ou généraux à confronter, voire à rectifier en fonction des connaissances acquises sur l'effet de l'inégalité des trames communales.

A. Robert (1975) a ainsi repéré des seuils d'équipement commercial dans les communes du Doubs. Les travaux de Calmes et al., (1979 et 1981) proposent une étude minutieuse de seuils significatifs de population communale, qui ne mesurent donc pas un marché desservi, mais la taille des communes d'implantation, et une explication des régularités et des variations observées. Les variations de seuils résultent de l'intervention complexe de la densité de la population, de la forme de l'habitat, du maillage communal, du degré d'urbanisation: contrairement à des affirmations antérieures, à volume égal, le degré de dispersion de l'habitat contribue à relever les seuils d'apparition des commerces; un peuplement rural a petite unité communale tend à les abaisser. En somme, une certaine élasticité joue, telle que les disparités de distance entre centres potentiels sont partiellement compensées par l'abaissement ou le relèvement des seuils. Des discontinuités se révelent toutefois.

Elles peuvent être utilisées dans la pratique et même être proposées comme seuil de ruralité. Ainsi, critiquant après tant d'autres l'arbitraire des seuils de population usuels, l'équipe de A. Piatier (1979) a proposé d'utiliser les discontinuités révélées par l'analyse quantitative de la dotation en services et de l'attractivité des communes françaises, pour distinguer entre rural et urbain. 
3. Géographie rurale et analyse spatiale: distributions spatiales, logiques de localisation et modèles géographiques

L'analyse qui précède a été centrée sur le contenu des questions de géographie rurale que les "quantitativistes" ont privilégiées. Un fil directeur les relie: le souci de décrire et d'interpréter les distributions spatiales ou la territorialité des phénomènes. En reprenant synthétiquement les propriétés de leurs démarches et la manière dont ils ont utilisé les outils, nous tenterons d'apprécier leur apport spécifique à la géographie rurale.

\subsection{Les résultats d'une double exigence d'explicitation}

L'exigence d'explicitation provient d'une contrainte machine: l'obligation de transformer les termes de l'analyse en descripteurs numérotés et en unités territoriales également numérotées! Elle a aussi été une exigence intellectuelle incluse dans le modèle de scientificité présenté au début. Cette double exigence conduit à trois types d'avantages potenticls.

Les exigences instrumentales et intellectuelles, alliées au traitement des données, aboutissent à faire progresser la réflexion sur l'adéquation entre concepts et objets de mesure. Certes la critique des sources et la sélection argumentée des variables sont loin d'être toujours faites. L'abondance des données de recensement a aussi incliné à une certaine facilité, en privilégiant leur emploi aux dépens de la construction de variables plus adéquates. Par opposition, les chercheurs travaillant sur les domaines mal saisis par l'information statistique ou ayant des données "qualitatives" sont handicapés pour utiliser l'arsenal actuel (pour l'agro-alimentaire par exemple). Mais, contrairement à ce qui est parfois écrit, l'usage d'un grand nombre de mesures pour cerner un phénomène oblige rapidement à dépister le sens latent des indices utilisés. Ainsi, les mesures de distances (groupe Dupont, 1975) ou de densités, essentielles pour caractériser les espaces ruraux, ne confondent guère sous les mêmes valeurs numériques des faits géographiquement différents: la diversité des types obtenus - ou la difficulté d'interprétation - renvoie en général à l'hétérogénéité que la mesure avait laminée, et conduit à des recherches nouvelles, vers d'autres métriques ou vers des pondérations plus adaptées aux faits à mesurer. La "réalité " des données apparaît de ce fait beaucoup moins évidente...

L'exigence d'explicitation, alliée à la recherche des régularités et au souci de préciser les ensembles spatiaux auxquels se rapportent les résultats, aboutit à une meilleure maîtrise des interdépendances ; 
cela s'est aussi traduit dans le choix des territoires d'étude et dans une attention particulière au territoire national. Celui-ci, précédemment territoire des synthèses est devenu un niveau opératoire de recherche (Béguin, 1974; Bonnamour, 1980; Aitchison et al., 1981...). Bien sûr la problématique «quantitative " n'est pas seule à intervenir; la conscience de l'interdépendance généralisée des évolutions des lieux les uns par rapport aux autres et le rôle croissant de la planification territoriale $y$ portent tout autant. C'est cependant grâce à cette perspective "quantitative " que l'on avance sur le lien entre un degré d'explication et le niveau d'observation, sur la portée de l'explication à l'intérieur de l'échelle des espaces. Poursuivie, l'étude régionale est alors davantage conduite comme l'étude d'un cas dans une série encore mal connue, que comme l'étude en soi d'un territoire particulier. Cette perspective d'analyse ne porte ni à éliminer l'événement isolé ni à obtenir absolument des lois universelles; elle cherche d'abord à préciser les règles du relatif en matière d'organisation territoriale et d'articulation des espaces entre eux. C'est en ce sens que l'on peut interpréter la liberté avec laquelle certains travaux ont repris comme territoire d'étude des régions récemment analysées (cf. le Languedoc viticole, le Berry; l'étude des marchés fonciers départementaux de la Mayenne par J. Macé et du Cher par V. Rey illustrant cette différence de perspective). La contrepartie de ce souci est un certain abandon de la dimension historique et de la reconstitution génétique des enchaînements particuliers.

Par ailleurs, l'analyse systématique de la signification des niveaux d'analyse et de l'effet d'agrégation des données dans l'explication géographique n'est pas encore entreprise. Des études portant sur des niveaux départemental, cantonal et communal (Aitchison, 1981 ; Rey, 1980) mettent en évidence aspects invariants et aspects spécifiques des phénomènes observés.

Un troisième apport lié à ce souci d'explicitation est celui de la transparence et de la reproduction (théoriques) des démarches. Malgré certaines difficultés de lecture des textes "quantitatifs", il devient cependant possible de contrôler les opérations de la démonstration et de l'interprétation, de reproduire et vérifier la validité des conclusions, de tester selon les mêmes méthodes des hypothèses: les conditions de la comparaison scientifique sont alors acquises. Le contenu des typologies rurales nous semblent avoir beaucoup gagné à cette clarté. L'étude avertie de $\mathrm{R}$. Chapuis peut servir d'exemple: elle contient les éléments de critique et même l'auto-critique de l'analyse des communes du Doubs en terme "d'intégration". De même les choix en matière de géographie agricole sont devenus plus clairs: l'explicitation des démarches a rendu nette l'opposition, qui 
semble aujourd'hui évidente, entre la tendance à traiter d'un groupe d'indicateurs considérés comme formant systèmes en eux-mêmes (telle l'analyse des systèmes d'exploitation) et la tendance à traiter séparément des éléments d'un complexe (Nicolas-Obadia, 1977). Cette alternative traduit le choix d'une interprétation en termes de fonctionnement spatialisé d'une activité, ou au contraire en termes de structures territoriales et d'individualisations régionales partiellement révélées à travers une activité.

\subsection{Bilan des procédures statistiques sélectionnées par les ruralistes quantitativistes}

Bien qu'utilisant une large gamme de techniques, les recherches de géographie quantitative rurale n'ont pas, jusqu'à présent, exploité toutes les possibilités de l'analyse des données, ni les potentialités de branches mathématiques (probabilités, théorie des graphes), ni celles des techniques d'analyse spatiale. En outre, des connaissances générales comme celles de la non-linéarité des relations liées à la distance, ou des convictions particulièrement ancrées chez les ruralistes de la "complexité " des phénomènes, sont peu explorées par les techniques appropriées que pourraient être la recherche d'ajustements non linéaires, la mesure des corrélations multiples, ou l'analyse systémique...

$\mathrm{La}$ majorité des travaux reste fondéc sur l'hypothèse de linéarité des relations entre les variables étudiées. L'étude des graphes de corrélation incite à s'interroger sur la nature des relations observées : représentent-elles des combinaisons fortes de variables, de type causal, ou de simples conjonctions territoriales (Calmès et al., 1978)? Plus sophistiqués mais reposant sur les mêmes principes sont les résultats d'analyses factorielles et de classifications automatiques. La cartographie des scores factoriels est largement usitée; mais elle suscite des comparaisons visuelles et des essais d'explications intuitifs des localisations, plus que des confrontations systématiques à d'autres variables (ex. Chapuis, 1979; Calmès et al., 1978, Auriac et al., 1974). Les analyses qui cherchent à formaliser plus étroitement une chaîne relationnelle entre variables sont peu nombreuses. Il en est ainsi pour la recherche dans le domaine des relations non linéaires et pour les analyses de corrélation et de régression multiples (sauf Rey, 1980, qui étudie les variations temporelles de catégories de taille d'exploitations et le marché foncier; Beguin, 1974; Durand-Dastès, 1981).

Pour ce qui est des statistiques "spatiales", les études d'autocorrélation spatiale, mesurant la ressemblance entre valeurs d'une variable dans les unités spatiales et leurs unités contiguës (cette ressemblance résultant soit de la dépendance des unités entre elles, soit de l'action des mêmes facteurs sur un ensemble d'unités proches) sont encore pionnières dans la recherche rurale (Durand-Dastès, 1981; Chesnais, 1981). De même les tests probabilistes destinés à juger du caractère (régulier, aléatoire, concentré...) d'une 
répartition spatiale sont peu diffusés (Baker, 1968; Calmès et al., 1981; Cauvin, Rimbert, 1976; Johnston, 1972 et Perry, 1977 sur la forme des aires matrimoniales - rare exemple d'études de flux de diffusion). Enfin les techniques de découpage utilisant des contraintes de contiguïté ne sont pas utilisées.

Ajoutons que l'usage des techniques cartographiques récentes: lissage des cartes, réalisation des surfaces de tendance... lesquelles semblent particulierement appropriées à l'étude d'un espace a priori continu et à des faits de contiguïté spatiale, est peu diffusé. Est-ce l'obstacle du "concret" et de la "spécificité des lieux" qui s'interpose d'une façon latente, ou seulement le caractère récent des techniques? (voir Béguin, 1974: cartes de densité et de potentiel de faits ruraux au Maroc; Guermond, 1977...).

Le constat qui ressort de ces choix est celui d'une incomplète maîtrise des techniques existantes et d'une attention trop timide à l'intégration de certains acquis. L'effectif des chercheurs qui ont fait l'effort de s'initier suffirait à expliquer ces lacunes; n'exprimentelles pas, en outre, leur difficulté à dépasser l'analyse des données, à passer de l'analyse des géométries spatiales à celles des mécanismes spatiaux? Plus généralement, ne reflètent-elles pas le peu de place prise par le test, le modèle spatial et la théorisation?

\subsection{Place des modèles et des théories spatiales}

On peut se demander si l'emploi de ces nouveaux outils a aussi fait progresser les connaissances théoriques. Les géographes ruralistes ont-ils avancé sur les problématiques classiques de la géographie rurale française, sur les interrogations théoriques relatives à la différenciation géographique et aux contraintes spatiales, ont-ils testé la pertinence des modèles de localisation et d'interaction diffusés pendant la période? La valeur ajoutée n'est pas aisément identifiable, ne serait-ce qu'en raison de la faible explicitation des questions antérieures et de la faible formalisation des méga-modèles préexistants, tels ceux des rapports ville-campagne, qu'ils soient d'inspiration marxiste ou culturaliste (P. George, E. Juillard).

On peut dire que malgré leur emploi d'outils quantitatifs, les ruralistes montrent une certaine réserve à «tronquer» le réel, acquis qu'ils sont à cette exigence aiguë de la complexité territoriale. On peut constater aussi que les modèles analytiques simples (gravitation, taille, distance...) sont plus facilement utilisés pour les études des espaces ruraux que pour les questions agricoles. Le manque de connaissances sur l'espace rural rend plus directe la confrontation avec des modèles. Le jugement caustique sur le schématisme et sur l'irréalisme des anneaux thünetiens expliquant l'organisation des campagnes à l'époque des transports rapides bloque toute tentative de test du modèle Von Thünen (à l'exception 
de Robert, 1974). Signalons certains apports. C'est ainsi qu'ont été démontrés le poids structurel de la taille des communes rurales (taille démographique et territoriale), celui de la distance à la ville (Chapuis, 1979; Groupe Dupont, 1975; et pour le péri-urbain: Chesnais, 1981; Groupe Dupont, 1981; Rolland-May, 1978). Néanmoins, les essais de mesure systématique de la "distance à la ville " (Groupe Dupont, 1975) achoppent sur les problèmes techniques d'analyse et sur la difficulté de comparer des distances à des centres de niveau différent. Par ailleurs les analyses de flux et de dotations ont montré des régularités qui confirment la réalité des organisations spatiales décrites par le modèle de Christaller, et infirment la conviction d'une anarchie des flux spécifique à l'espace rural.

Il se dessine des tentatives qui se veulent une contribution à la théorisation de la géographie. G. Nicolas-Obadia (1977) a proposé une axiomatisation de la géographie, sur la base de ses études théoriques et empiriques sur la différenciation de l'espace agricole. Relevant de familles idéologiques diverses, d'autres auteurs ont également cherché à fournir des interprétations théoriques. Elles se révèlent plus réalistes que les modèles "anglo-saxons" basés sur les hypothèses de l'homo aeconomicus aux dépens des théories sociales fondées sur le conflit (... effet sans doute de patrimoine scientifique); elles se révèlent aussi plus engagées à débattre des composantes territoriales des systèmes étudiés que les approches classiques. Y. Guermond (1977) s'est attaché à la définition des règles de combinaison des éléments agricoles susceptibles de décrire un espace en phase de changement; sa thèse conclut à l'inexistence de telles règles, tout comme à la quasi-impossibilité de définir les interactions entre différenciation spatiale et différenciation sociale. La thèse de F. Auriac (1979) offre des conclusions apparemment plus positives; parti de l'interprétation selon laquelle le vignoble languedocien est une création des villes bourgeoises, il réinterprète les différentes phases de vitalité et de crise du vignoble en termes de fonctionnement systémique (culture pérenne — main-d'œuvre coopérative de vinification) et propose de reconnaître un système viticole languedocien dont l'identité et la permanence seraient moins appuyées sur les noyaux urbains que sur les multiples centres locaux de coopérative; cette interprétation fait alors intervenir non seulement les rapports de production mais aussi les trames spatiales nées du vignoble. L'identification du "facteur» spatial dans le système est alors la contribution originale de l'étude.

Reprenant la question des structures foncières agricoles, V. Rey (1980) analyse les configurations spatiales mouvantes liées à la concentration foncière et à l'évolution des régimes juridiques en termes de saturation spatiale et de rente foncière. Elle propose pour 
ce concept, déjà riche d'applications multiples, un autre usage géographique. Elle le définit comme une combinaison de quatre composantes (rente de fertilité, rente de situation relative, rente d'inégalité de moyens, rente juridique); l'efficacité pour l'analyse géographique n'est acquise qu'à travers l'interaction des quatre composantes qui ne sont pas dans un rapport hiérarchique stable entre elles. Reste, dans cette perspective, à construire un modèle territorial d'évolution des systèmes fonciers agricoles où le degré de saturation de l'espace soit élément du système à l'égal des contraintes technico-économiques!

La seule tentative de modélisation systémique porte sur l'espace rural (Durand, 1981). Le modèle AMORAL explore la dynamique de la cellule rurale locale et teste par scénario les effets probables d'interventions extérieures d'aménagement. Cet exemple témoigne de l'interdépendance entre recherche théorique et applications pratiques. La formalisation obligée qu'impose la démarche modélisante (avec contrainte de vérification des hypothèses) est certes fort difficile, mais elle permet de découvrir les points faibles de notre connaissance.

\section{Conclusion}

C'est l'image de la spirale de progression de la connaissance géographique que nous retiendrons au terme de cette analyse des débuts de la géographie "quantitative et théorique" dans le domaine rural. La rapidité de l'outil a rendu possible le rêve de va-et-vient entre les informations et les hypothèses. Toutefois la méthode itérative se révèle réellement efficace à l'intérieur de démarches critiques, qui associent une analyse des manifestations territoriales des phénomènes à une formulation en termes de régularités et de modèles de fonctionnement.

Deux conclusions nous paraissent plus spécifiques à la géographie rurale. Une certaine réticence à l'égard de ces démarches n'est-elle pas la manifestation d'une réticence plus générale des géographes ruralistes à l'égard des débats d'idées et des confrontations de thèses, entre eux ou avec les autres spécialistes des campagnes? La différence d'approche selon qu'il s'agit du domaine agricole ou du domaine rural n'est-elle pas le reflet de l'inégal avancement de la connaissance sur ces domaines? 


\section{Bibliographie}

Après un état des travaux cernant le champ de la géographie rurale française, les titres ont été séparés entre géographie agricole et géographie rurale, et ils ont été présentés selon l'ordre de parution.

La sélection a retenu les travaux reposant sur un outil informatique et une analyse statistique, ainsi que ceux dont les titres se réfèrent explicitement à la théorie.

On a dépouillé systématiquement une large gamme de revues nationales. L'Espace géographique, les Annales de Géographie, le Bulletin de l'Association des Géographes français, Hérodote ainsi que Etudes rurales et Economie rurale. Cette information a été complétée par le recours aux publications les plus ouvertes aux recherches "quantitatives et théoriques": Cahiers de Geographie de Besançon (Séminaires et Notes de Recherche, qui contiennent les Actes des colloques annuels de Besançon), Brouillons Dupont, Travaux de l'Institut de Géographie de Reims... et aux autres revues dont des références étaient signalées.

1. État des travaux

France

Berger M., Bontron J.-C., Mathieu N., Peyon J.-P., Plet F., Robic M.-C., 1975, "Eléments de réflexion sur l'aménagement de l'espace rural en France", Réflexions sur l'espace rural français. Approches, définitions, aménagement. Université de Paris I, Ecole Normale supérieure de Fontenay-aux-Rose, p. 53-85.

Berger M., Gillette C., Robic M.-C., 1975, "L'étude des espaces ruraux français à travers trois quarts de siècle de recherche géographique: l'exemple des thèses de Doctorat d'Etat", Réflexions sur l'espace rural français. Approches, définitions, aménagement. Université de Paris I, Ecole Normale supérieure de Fontenay-auxRoses, p. 3-51.

Berger M., Fruit J.-P., Plet F., Robic M.C., 1980, "Rurbanisation et analyse des espaces ruraux péri-urbains", L'Espace géographique, 4, p. 303-313.

Bonnamour J., 1973, Geographie rurale, méthodes et perspectives, Paris, Masson, 170 p.

Chiva I., Rambaud P., (éd.), 1972, Les Etudes rurales en France. Tendances et organisation de la recherche, Paris, La Haye, Mouton, 368 p.

Claval P., 1968, "Economie et géographie rurales. Chronique de géographie économique ", Revue de Géographie de l'Est, 1-2, p. 180-212.

Economie Rurale, 1977, "Répertoire des travaux actuels de sciences humaines dans le monde rural", Economie rurale, 3.

Flatrès P., 1968, "Les travaux français de géographie rurale de 1964 à 1968", Annales de Géographie, 424, p. 734-743.

Flatrès P., 1972 a, "La géographie rurale en France", in: Recherches géographiques en France, Montréal 1972, Paris, Comité national français de Géographie, p. 189-194.

Flatrès P., 1972 b, "Géographie agraire et aménagement rural : réflexions sur une évolution", in: La Pensée géographique française contemporaine (Mélanges offerts au Professeur A. Meynier), Saint-Brieuc, Presses Universitaires de Bretagne, p. $431-442$.

Juillard E., 1964, "Géographie rurale française. Tableaux récents (1957-1963) et tendances nouvelles", Etudes rurales, 13-14, p. 46-70.

Juillard E., Meynier A., de Planhol X., Sautter G., 1957, "Structures agraires et paysages ruraux. Un quart de siècle de recherches françaises", Annales de l'Est, $189 \mathrm{p}$.

Mathieu N., 1982, "Questions sur les types d'espaces ruraux en France", L'Espace géographique, 2, p. 95-110.

Peyon J.-P., Plet F., 1974, "L'aval de l'agriculture dans les publications géographiques françaises", in: Equipe de Géographie Rurale du Laboratoire Associé de Géographie Humaine, Rapport n², p. 125-156. 
Rey V., Jammes A.-M., 1974, "Les géographes ruraux et le milieu naturel ", in: Equipe de Géographie Rurale du Laboratoire Associé de Géographie Humaine, Rapport $\mathrm{n}^{\circ} 2$, p. 165-172.

Rey V., Sachet M., 1975, "Utilisation du sol, problèmes de sources", in: Réflexions sur l'espace rural français. Approches définitions, aménagement, Laboratoire de géographie rurale, université de Paris I, E.N.S., Fontenay-aux-Roses, 35 p.

Etranger

Berry B., 1971, Géographie des marchés et du commerce de détail, Paris, A. Colin, $255 \mathrm{p}$.

Cloke P.J., 1980, "New emphases for applied rural geography ", Progress in Human Geography, 2, p. 181-217.

Clout H., 1981, "Rural settlements", Progress in Human Geography, 3, p. 408-413.

Grigg D., 1981, "Agricultural geography», Progress in Human Geography, 2, p. 268276 .

Nitz H.-J., 1978, "Où en est la recherche en matière de géographie agraire en République Fédérale Allemande?», L'Espace géographique, 3, p. 199-207.

2. Ouvrages et articles ćtudiés (par ordre de parution)

Géographie agricole

Baker A.-R.-H., 1968, «Etablissements ruraux sur la marge sud-ouest du Bassin parisien dans les premières années du XIX ${ }^{*}$ siècle. Description analytique des types », Norois, 60, p. 481-492.

Chiffre J., 1969, "Une nouvelle formule de dispersion de l'habitat rural: son application au Nivernais", Revue de Géographie de l'Est, janv.-juin, p. 149-175.

Gutelman M., 1971, «Description des structures agraires et formalisation des rapports sociaux en agriculture», Etudes rurales, 41, p. 15-48.

Nicolas-Obadia G., 1971, «La carte statistique agricole: son commentaire et son utilisation", Etudes rurales, 43-44, p. 7-77.

Bertrand G., 1972, «Ecologie d'un espace géographique : les géosystèmes du Valle de Prioro (Espagne N.-O.)", L'espace géographique, p. 113-128.

Bonnamour J., Gillette C., Guermond Y., 1972, "Les systèmes régionaux d'exploitation agricole en France, méthode d'analyse typologique ", Etudes rurales, 43-44, p. $98-169$.

Drain M., 1972, "Le géographe et la programmation linéaire ", in: Etude géographique des exploitations agricoles, Cahier de l'Equipe rurale du LA 142, p.135-139.

LA 142, Equipe Rurale, 1972, "Etude géographique des exploitations agricoles. Réflexions et propositions méthodologiques», Université Paris I, ronéo, en particulier trois articles sur la programmation linéaire.

Briyant C.-R., 1973, "Urbanisation et structures agricoles dans la région parisienne entre 1955 et 1966 ", Etudes rurales, 49-50, p. 205-244.

Dumolard P., 1973, "Disparités spatiales de l'intensivité agricole en Italie ", Espace géographique, 1 , p. 50-62.

Guermond Y., 1973, “Prospection de techniques d'analyses des données en géographie agricole", Centre de Recherches sur l'évolution de la vie rurale, Caen, Publications $\mathrm{n}^{\circ} 2$, p. 291-316.

Guermond Y., Massias J.-P., 1973, "L'utilisation agricole du sol en France: comparaison de deux méthodes de traitement de l'information", Espace géographique, t. 2, 4, p. 267-273.

Béguin H., 1974, L'Organisation de l'espace au Maroc, Bruxelles, 780 p.

Béguin H., 1974, "Densité de population, productivité et développement agricole (au Maroc)", Espace géographique, 3, p. 273-286.

Boichard J., 1974, "Systèmes agricoles et structures d'exploitation en France», Cahiers de géographie de Besançon, 23, p. 89-163. 
Nicolas-Obadia G., 1974, Atlas statistique agricole vaudois, Lausanne, Cahier de l'Aménagement régional, $\mathrm{n}^{\circ} 16$.

Guermond Y., Leduc A., 1975, "Réflexions sur les procédures de classification automatique ", Espace géographique, 25, p. 193-204.

Guermond Y., 1975, «Deux programmes de simulation... sur la diffusion des stabulations libres en Normandie et sur l'évolution communale des combinaisons culturales en Normandie ", Cahiers de géographie de Rouen, 4, p. 21-48.

Lenco M., 1975, "Nouvelle classification des exploitations agricoles", Etudes rurales, 57, p. 7-35.

Uzant Lepagnot L., 1975, "Un espace agricole est-il un espace atone?", B.A.G.F., $n^{\circ} 432$, p. 55-62.

Beguin H., 1976, "The Global Evaluation of a Geographic Environment: a Contribution to Geographic Theory ", L'Espace géographique, 3, p. 177-180.

Maurel M.-C., 1976, "Différenciation spatiale des unités de production agricoles en Russie d'Europe ", Annales de Géographie, p. 694-724.

Deffontaines J.-P., Osty P.-L., 1977 "Des systèmes de production agricole aux systèmes agraires ", L'Espace géographique, 3, p. 195-199.

Lazarev G., 1977, "Une analyse régionale du problème agraire en Colombie», L'Espace geographique, 2, p. 113-131.

Nicolas-Obadia G., 1977 "La théorie des noyaux agricoles », L'Espace géographique, 1, p. 25-39.

Barre A., Dion R., 1978, "La transition Boulonnais-Montreuillois, un exemple de l'emploi de techniques quantitatives à la connaissance du milieu agricole", Hommes et Terres du Nord, 1, p. 61-89.

Bertrand G., 1978, "Le paysage entre nature et société ", Revue de Géographie des Pyrénées et du Sud-Ouest, 49, 2, p. 239-258.

Calmès R., 1978, Développement et progrès agricoles dans les Segalas et Levezou, Thèse de doctorat, université de Paris VII, 2 vol., $579 \mathrm{p}$.

Gilg J.-P., 1978 "Photographies aériennes et espace rural », Etudes rurales, 71-72, p. 274-316.

Moindrot C., 1978, "Le prix de la terre agricole aux Etats-Unis", Brouillons Dupont, 3, p. $5-25$.

Auriac F., 1979, Système économique et espace. Un exemple en Languedoc, Thèse de doctorat, université P. Valéry, Monptellier.

Brunet-Le Rouzic L., 1979 "L'analyse comparative des structures d'exploitation", Travaux de l'Institut de Géographie de Reims, 37, p. 55-57.

Charles J., 1979, "Approche quantitative de l'espace agricole basco-béarnais", Travaux de l'Institut de Géographie de Reims, 37, p. 3-25.

Cote M., 1979, "Le produit agricole algérien", L'Espace géographique, p. 143-152.

Garcia Ramon M., 1979, "Une interprétation du paysage agraire; le cadre théorique et sa vérification empirique ", Brouillons Dupont, 4, p. 5-14.

Guermond Y., 1979, "Sorties graphiques et structures spatiales: l'exemple de l'espace agricole français", Cahiers de Géographie de Rouen, p. 10-11.

Guermond Y., 1979, Le Système de différenciation spatiale en agriculture. La France de l'Ouest de 1950 à 1975, Thèse, atelier de reproduction de Lille, $370 \mathrm{p}$.

Bonnamour J., Gillette C., 1980, Les Types d'agriculture en France, 1970, essai méthodologique, Paris, C.N.R.S., 180 p.

Rey V., 1980, L'Agrandissement spatial des exploitations, Thèse de doctorat, université de Paris I, $520 \mathrm{p}$.

Brossard T., Wieber J.-C., 1980, "Essai de formulation systémique d'un mode d'approche du paysage ", Bulletin de l'Association des Géographes Français, 468, p. 103-118.

Wieber J.-C., 1980, Etablissement d'un modèle régional de classification typologique des paysages, Bulletin de l'Association des Géographes Français, 468, p. 125-129. 
Moindrot C., 1981, Le Modèle agricole de J.-H. von Thünen et ses applications, polycopié, université Paris VII, $160 \mathrm{p}$.

Rey V., 1981, "Foin de fichiers mal fichus... et quelques conclusions sur le besoin de terre, obtenues par l'analyse des données ", Informatique et Sciences humaines, 51, p. 69-81.

Sanders L., 1981, "L'utilisation agricole du sol en France: composantes régionales et sociales", Informatique et Sciences humaines, 51, p. 23-45.

Rey V., 1982, Besoin de terre des agriculteurs, Paris, Economica, 380 p.

Géographie rurale

Loux F., de Virville M., 1969 "Le système social d'une région rurale: le Châtillonnais », Etudes rurales, 35, p. 5-135.

Johnston R.-J., Perry P.-J., 1972, "Déviation directionnelle dans les aires de contact. Deux exemples de relations matrimoniales dans la France rurale du XIX " siècle", Etudes rurales, 46, p. 23-33.

Chapuis R., 1973, "De l'espace rural à l'espace urbain. Problèmes de typologie", Etudes rurales, 49-50, p. 122-136.

Auriac F., Bernard M.-C., 1974, "Composantes et types socio-professionnels des campagnes du Languedoc-Roussillon", Bulletin de la Société languedocienne de Géographie, 1, p. 3-20.

Béguin H., 1974, L'Organisation de l'espace au Maroc, Bruxelles, Académie royale des Sciences d'Outre-Mer, $787 \mathrm{p}$.

Calmès R., Moindrot C., Peyon J.-P., Plessis-Fressard M., 1974, "La population rurale en France. Approche quantitative", Travaux du Laboratoire d'Analyse spatiale des données géographiques, université Paris VII, p. 1-26.

Chapuis R., 1974, "Essai de typologie factorielle sur un échantillon de cent communes rurales du Doubs", Séminaires et notes de recherche $n^{\circ} 11$. Cahiers de Géographie de Besançon, p. 69-128.

Fontaine J., 1974, "Les loisirs des jeunes dans le district rural de Montrevel-enBresse. Les apports de l'analyse factorielle". Séminaires et notes de recherche $n^{\prime 11}$. Cahiers de Géographie de Besançon, p. 129-170.

Robert A., 1974, "Réflexions pour une étude théorique de la localisation des résidences secondaires ", Cahiers de Géographie de Besançon, 23, p. 165-208.

Auriac F., Bernard M.-C., Lochard E., 1975, "Le changement social dans les campagnes languedociennes", L'Espace géographique, 4, p. 239-250.

Bailly A.-S., Varacca C., 1975, "Lieux centraux et comportements commerciaux: étude des niveaux inférieurs de la hiérarchie", Cahiers de Géographie de Besançon, 24, p. 191-220.

Groupe Dupont, 1975 a, "La distance à la ville: essais d'analyses factorielles appliquées aux cas de Grenoble et de Montpellier", L'Espace géographique, 4, p. 225-238.

Groupe Dupont, 1975 b, "Réflexions critiques sur l'analyse des composantes principales ", Séminaires et notes de recherche $n^{\circ} 12$, Cahiers de Géographie de Besançon, p. 145-171.

Limouzin P., 1975, "Analyse du dynamisme communal en milieu rural. Le cas de la Touraine ", L'Espace géographique, 4, p. 251-258.

Robert A., 1975, "Les hiérarchies du monde rural: centres ruraux et de service en Franche-Comté ", Cahiers de Géographie de Besançon, p. 149-189.

Cauvin C., Rimbert S., 1976, La Lecture numérique des cartes thématiques, Fribourg, Editions universitaires de Fribourg, $172 \mathrm{p}$.

Guigou J.-L., 1976, "Les relations intercommunales et l'organisation de l'espace socio-économique", Séminaires et Notes de Recherche n"15-16, Cahiers de Géographie de Besançon, p. 71-104.

Bontron J.-C., Mathieu N., 1977, La France des faibles densités. Délimitation, problemes, typologies, Paris, A.C.E.A.R., 170 p. (2 éd.) dont: Aitchison J.-W., 
"Contrôle des découpages proposés par une classification ascendante hiérarchique », p. 161-170.

Chapuis R., 1977, "Typologie des communes de moins de 5000 habitants du département du Doubs", Communication aux Journées de Géographie rurale, Amiens, $11 \mathrm{p}$.

Perry P.-J., 1977, "Mariage et distance dans le canton du Bleymard (Lozère), 1811-1820 et 1891-1900", Etudes rurales, 67, p. 61-70.

Auriac F., Bruynooghe M., 1978, "Une classification sur plus de 1500 communes: la distance à la ville en Languedoc-Roussillon". Séminaires et notes de recherche $n^{\circ}$ 17, Cahiers de Géographie de Besançon, p. 207-217.

Calmès R., Delamarre A., Durand-Dastès F., Gras J., Peyon J.-P., 1978, L'Espace rural français, Paris, Masson, $175 \mathrm{p}$. + planches.

Cheylan J.-P., Farinas Del Cerro L., Desbordes-Cheylan F., 1978, Mécanismes de la croissance péri-urbaine méridionale: Martigues. Une méthode d'analyse formelle, G.A.M.S.A.U., L.I.S.H.-C.N.R.S., 281 p.

Rolland-May C., 1978, "L'urbanisation du pays messin", L'Espace géographique, 1, p. 43-51.

Calmès R., Delamarre A., Durand-Dastès F., Gras J., Peyon J.-P., 1979, « Les réseaux du commerce de détail dans le Gers", Revue géographique des Pyrénées et du Sud-Ouest, 50, 3, p. 407-444.

Calmès R., Delamarre A., Durand-Dastès F., Gras J., Peyon J. P., 1979 a, "Le fait communal en France", Espace 90, numéro spécial, 63 p.

Chapuis R., 1979, Espace et société. Géographie sociologique des campagnes du département du Doubs, Thèse de doctorat d'Etat, université Louis Pasteur, Strasbourg, 3 t., $1133 \mathrm{p}$.

Limouzin P., 1979, Le Dynamisme des communes rurales françaises, Thèse de doctorat d'Etat, université de Paris I, Paris.

Piatier A. (sous la direction de), 1979, Radioscopie des communes de France. Ruralite et relations villes-campagnes. Une recherche pour l'action, Paris, Economica, $549 \mathrm{p}$.

Renard J.-P., 1979, "Structures d'un espace rural: le Ternois", Travaux de l'Institut de Géographie de Reims, 37, p. 27-43.

Groupe Dupont, 1980, Montpellier 1954-1978; processus de transformation de l'espace et développement du bâti urbain. - Montpellier - Caen, 238 p. + H.T.

Maurel M.-C., 1980, La Campagne collectivisée. Société et espace rural en Russie, Paris, Anthropos, $300 \mathrm{p}$.

Pinchemel P., 1980, La France. Milieux naturels, populations, politiques, Paris, A. Colin, t. II, $410 \mathrm{p}$.

Aitchison J.-W., Bontron J.-C., Velard L., Brochot A., 1981, "L'avenir des zones à faibles densité", in: La France rurale, images et prospectives, Travaux et recherches de prospective, Paris, La Documentation française, p. 81-160.

Bernard M.-C., 1981, "Essais d'analyses diachroniques: exemples de changements dans les campagnes languedociennes", Informatique et Sciences humaines, 51, p. 47-67.

Calmès R., Delamarre A., Durand-Dastès F., Gras J., 1981, "Etudes sur les réseaux de commerces élémentaires fixes en France" (avec référence particulière à l'espace rural), Travaux de l'Institut de Géographie de Reims, 48, $116 \mathrm{p}$.

Chesnais M., 1981 a, "Apprentissage des processus de transformation de l'espace par l'urbanisation", Informatique et Sciences humaines, 51, p. 13-21.

Chesnais M., 1981 b, "Le prix des terrains autour de Montpellier. Du constat à l'interprétation: effet de voisinage, effet de distance, autocorrélation statistique ou spatiale?". Travaux de l'Institut de Géographie de Reims, 47, p. 39-47.

Durand M.-G., 1981, "Un exemple de modélisation en géographie régionale: analyse systémique et dynamisation régionale dans les Préalpes du Sud. Le modèle A.M.O.R.A.L.", Séminaires et Notes de recherche $n^{\circ} 21$, Cahiers de Géographie de Besançon, p. 145-157. 
Durand-Dastès F., 1981, "Les actifs non agricoles des campagnes françaises. Corrélations et autocorrélations ", Travaux de l'Institut de Géographie de Reims, 47, p. 17-38.

Groupe Dupont, 1981, «Montpellier 1954-1978. Processus de transformation de l'espace et développement du bâti urbain ", Bulletin de la Société languedocienne de Géographie, 4, p. 259-295.

Chapuis R., 1982, Les Ruraux du département du Doubs. Eléments de géographie sociologique, Besançon, C.E.T.R.E., 388 p.

Résumé. - Au seuil de la modélisation systémique, les travaux de géographie rurale écrits dans une perspective "quantitative et théorique" sont peu nombreux. Leur unité ne tient pas au choix de sujets particuliers ou de techniques préférentielles, mais à une orientation de recherche. Celle-ci valorise l'explicitation, la mesure et vérification, les constructions théoriques. Les résultats tiennent compte des connaissances acquises, ce qui conduit à un effort particulier de théorisation dans le domaine agricole déjà très étudié, et à une exploration de la spécificité territoriale de cette nouvelle entité qu'est l'espace rural. Ce qui lie les deux domaines, c'est la préoccupation de la composante territoriale des phénomènes et un effort de conceptualisation proprement géographique. Ces démarches s'orientent vers les modeles spatiaux les plus connus, mais non retenus par la géographie rurale classique. La décennie 1980 s'ouvre sur une vision plus claire des points à approfondir.

Abstract. - On the fringe of systemic model-making, works done in rural geography with a theoric and quantitative approach are rather scarce. Their common factor has nothing to do with the choice of particular topics or techniques, but comes from the orientation of the research itself. The stress is put on explanations, quantitative measurements - and their checking - and theoretical models. Results take previous knowledge into account, which leads first to a special theorizing effort in the already much-studied agricultural field, and, second, to an attempt to define a new spatial entity, the so-called rural space. Both fields are linked by the concern for the spatial component of the phenomena and an effort of geographical conceptualization. These processes lead to the most well-known models, so far neglected by classic rural geographers. The eighties open out on a clearer view of the points that need to be deepened. 